\title{
ESTUDO DA AUDIÇÃO EM CRIANÇAS PORTADORAS DE DEFICIÊNCIA VISUAL
}

\author{
Study of hearing in children with visual deficiency
}

\author{
Elisandra dos Santos (1), Angélica Maria Bicudo Zeferino ${ }^{(2)}$, \\ Heloisa Gagheggi Ravanini Gardon Gagliardo ${ }^{(3)}$, Maria Francisca Colella-Santos ${ }^{(4)}$
}

\section{RESUMO}

Objetivo: analisar a audição de escolares portadores de deficiência visual (baixa visão e cegueira) e caracterizá-los quanto ao desempenho escolar. Método: anamnese e avaliação audiológica de 30 (100\%) crianças de 8 a 12 anos, sendo 15 (50\%) do Grupo I, constituído por portadores de deficiência visual e 15 (50\%) do Grupo II formado por crianças com visão normal, sem queixas auditivas e/ ou escolares. Foram realizadas meatoscopia, audiometria tonal liminar, logoaudiometria e imitanciometria; além dos testes de Localização Sonora em Cinco Direções, Memória Sequencial para Sons Verbais e Não-Verbais, Fala com Ruído, Dicótico de Dígitos, Padrão de Duração e Detecção de Intervalos Aleatórios (RGDT). Os pais e/ou responsáveis responderam questões sobre o histórico escolar. Resultados: na avaliação audiológica básica, no Grupo I, $04(26,6 \%)$ escolares obtiveram resultados alterados, sendo $02(13,3 \%)$ com perda auditiva do tipo condutiva, $01(6,8 \%)$ com perda auditiva sensórioneural e $01(6,8 \%)$ com perda mista. No Grupo II, todos os escolares apresentaram resultados normais. Nos testes do Processamento Auditivo, verificou-se que o Grupo I obteve resultados semelhantes ao Grupo II nos testes de Localização Sonora, Memória Seqüencial para Sons Não-Verbais e Fala com Ruído. Nos demais testes, O Grupo I mostrou desempenho inferior ao Grupo II. Conclusão: o Grupo I mostrou-se com desempenho desfavorável em relação ao Grupo II tanto na avaliação audiológica básica, como na bateria de testes do Processamento Auditivo. O mesmo foi observado para o desempenho escolar, havendo no Grupo I maior número de repetências e dificuldades no aprendizado da leitura e escrita.

DESCRITORES: Audição; Baixa Visão; Cegueira; Percepção Auditiva

(1) Fonoaudióloga; Professora do Centro Universitário de Araraquara, UNIARA, Araraquara, SP; Mestre em Saúde da Criança e do Adolescente pela Universidade Estadual de Campinas.

(2) Terapeuta ocupacional; Docente em Educação Especial e Reabilitação III do Centro de Estudos e Pesquisas em Reabilitação Professor Doutor Paulo O.S. Porto da Faculdade de Ciências Médicas da Universidade Estadual de Campinas, UNICAMP, Campinas, SP; Doutora em Ciências Médicas - Área de Concentração/Neurologia - pela Faculdade de Ciências Médicas da Universidade Estadual de Campinas.

(3) Médica Pediatra; Professora Livre-Docente da Faculdade de Ciências Médicas da Universidade Estadual de Campinas, UNICAMP, Campinas, SP; Doutora e Livre-Docente em Saúde da Criança e do Adolescente pelo Departamento de Pediatria da Faculdade de Ciências Médicas da Universidade Estadual de Campinas.

(4) Fonoaudióloga; Professora Doutora e Coordenadora do Curso de Fonoaudiologia do Centro de Estudos e Pesquisas em Reabilitação Professor Doutor Paulo O.S. Porto

\section{INTRODUÇAO}

A audição não é uma habilidade sensorial única, isolada. Não se refere a uma mera detecção do sinal acústico, uma vez que muitos mecanismos e processos neurofisiológicos e cognitivos são necessários para uma perfeita decodificação, percepção, reconhecimento e interpretação do sinal auditivo. O Sistema Nervoso Auditivo Central (SNAC) é, portanto, um sistema altamente complexo e redundante, constituído por múltiplos componentes e níveis de organização interativa seqüencial e

da Faculdade de Ciências Médicas da Universidade Estadual de Campinas, UNICAMP, Campinas, SP; Doutora em Ciências dos Distúrbios da Comunicação Humana pela Universidade Estadual de São Paulo.

Conflito de interesses: inexistente 
paralela 1 . Tem papel relevante e essencial para o correto reconhecimento e discriminação de eventos auditivos, desde os eventos mais simples, como um estímulo não verbal, até mensagens complexas, como é o caso da fala e da linguagem ${ }^{2,3,4}$.

Processamento Auditivo (PA) é aquilo que fazemos com o que ouvimos, ou seja, é a construção que se faz acerca do sinal auditivo para tornar a informação funcionalmente útil. Constitui-se numa série de operações mentais que o indivíduo realiza ao lidar com informações recebidas, via sentido da audição, e que dependem de uma capacidade biológica inata, do processo de maturação e das experiências e estímulos no meio acústico ${ }^{5,6}$. Para a $\mathrm{ASHA}^{1}$, o Processamento Auditivo se refere à eficiência e eficácia com que o SNAC utiliza a informação auditiva. Ele é responsável pelos seguintes fenômenos: localização e lateralização do som, discriminação auditiva, reconhecimento de padrões auditivos, aspectos temporais da audição e desempenho auditivo com sinais acústicos competitivos e degradados. Estes mecanismos e processos são supostamente aplicáveis em sinais não verbais bem como em verbais e afetam muitas áreas funcionais, incluindo fala e linguagem.

A avaliação do PA é constituída por testes especiais comportamentais ou eletrofisiológicos que avaliam as habilidades auditivas. É um procedimento útil para diagnosticar o uso funcional correto e eficiente da audição, possibilitando a detecção do Distúrbio do Processamento Auditivo e adequada intervenção, para que não ocorra um bloqueio sério na comunicação, aprendizagem e comunicação social $^{7}$

Embora o SNAC seja crítico para as funções auditivas, incluindo o processamento da linguagem falada e de muitos outros sinais complexos, diversos outros fatores também estão envolvidos. Mesmo a tarefa mais simples é influenciada por funções de alto nível e não específicas à modalidade, como atenção, a aprendizagem, a motivação, a memória, fenômenos que são, principalmente, mediados pela visão ${ }^{8}$

Crianças portadoras de deficiência visual (cegueira e baixa visão), por sofrerem uma privação do apoio visual, para a captação das redundâncias extrínsecas da mensagem, como visualização da comunicação gestual e leitura orofacial, poderão vir a apresentar diferenças de desempenho nas habilidades auditivas envolvidas no Processamento Auditivo. O desenvolvimento normal das habilidades auditivas nestas crianças é indispensável pelo fato da audição tornar-se, juntamente aos outros sentidos remanescentes, as principais via de acesso às informações e aprendizagem ${ }^{8}$
Conhecer os reais parâmetros do processamento auditivo de deficientes visuais torna-se fundamental para que seja possível orientar familiares, cuidadores e professores quanto ao desenvolvimento global, aprendizagem e socialização desses indivíduos.

Desta forma, o objetivo deste trabalho foi analisar a audição de escolares portadores de deficiência visual (baixa visão e cegueira). Além disso, caracterizá-los quanto ao desempenho escolar.

\section{MÉTODO}

Trata-se de um estudo de corte transversal, clínico e comparativo, realizado no Centro de Estudos e Pesquisas em Reabilitação - Prof. Dr. Gabriel O. S. Porto - CEPRE/FCM/UNICAMP.

Foram avaliadas 30 crianças, dos gêneros masculino e feminino, na faixa etária entre 08 e 12 anos. Assim, de acordo com os critérios de inclusão estabelecidos, formaram-se dois grupos, pareados de acordo com o gênero e a idade, a saber:

- Grupo I: Grupo de estudo, composto por 15 escolares portadores de Deficiência Visual cuja deficiência foi classificada segundo a escala Optométrica de Snellen ${ }^{9}$ em Baixa Visão Severa, Baixa Visão Profunda, Próximo a Cegueira e Cegueira. Não apresentavam comprometimentos do desempenho cognitivo, como deficiência mental entre outros transtornos e eram participantes de programas de reabilitação de instituições do Estado de São Paulo, além de estarem inseridas em escola regular.

- Grupo II: Grupo controle, formado por 15 escolares com visão normal, sem queixas escolares e/ou auditivas.

Vale ressaltar que, como a grande maioria das crianças usuárias dos programas de reabilitação para Deficientes Visuais das instituições participantes apresentavam comprometimentos associados à deficiência visual, estas foram excluídas, restringindo o número de participantes da amostra.

A coleta de dados foi realizada em duas etapas: Primeira etapa: envolveu a anamnese e a avaliação audiológica básica dos 30 escolares da amostra. A anamnese foi direcionada aos pais e/ ou responsáveis e constituída por perguntas relacionadas ao histórico e desempenho escolar e aos sinais e sintomas sugestivos de alterações auditivas periféricas e/ou centrais. A avaliação audiológica básica foi composta por meatoscopia, imitanciometria (timpanometria e pesquisa dos reflexos acústicos contralaterais e ipsilaterais), audiometria tonal liminar, logoaudiometria (Limiar de Reconhecimento de Fala - LRF e Índice de Reconhecimento 
de Fala - IRF). Considerou-se como critérios de normalidade para esta etapa, média de audibilidade para as frequências de 500, 1000 e $2000 \mathrm{~Hz}$ maior ou igual a $25 \mathrm{~dB} N A$; IRF maior ou igual a $88 \%$, curva timpanométrica tipo $A$ e presença de reflexos acústicos contralaterais e ipsilaterais ${ }^{10}$. No caso de resultados compatíveis com perda auditiva, essas foram classificadas quanto ao tipo em condutiva, sensórioneural ou mista ${ }^{10}$.

Participaram da segunda etapa de avaliação, as crianças que obtiveram média de audibilidade para tom puro nas frequências de 500, 1000 e $2000 \mathrm{~Hz}$ menor que $40 \mathrm{~dB} N A$, simétrica preferencialmente, com Índice de Reconhecimento de Fala de no mínimo $70 \%$ de acertos em cada orelha e diferença entre as orelhas não excedendo $20 \%{ }^{7}$. Apenas a criança número 3 do Grupo I não participou desta etapa, pois os resultados obtidos não estavam de acordo com os critérios estabelecidos.

Vale ressaltar que além da análise comparativa entre os grupos, objetivamos analisar também o desempenho auditivo individual dos participantes de acordo com os critérios de normalidade para crianças sem queixas escolares e/ou auditivas, propostos na literatura.

Segunda etapa: foram aplicados os testes de avaliação do Processamento Auditivo, sendo Teste de Localização Sonora em Cinco Direções, Memória Seqüencial para Sons Verbais e Não Verbais, Fala com Ruído e Dicótico de Dígitos ${ }^{7}$, além dos testes Padrão de Duração'11 e Detecção de Intervalos Aleatórios (RGDT) ${ }^{12}$. Os critérios de normalidade adotados foram de acordo com a padronização proposta pelo autor de cada teste ${ }^{7,12,13}$.

Foram considerados como portadores de distúrbio do Processamento Auditivo os indivíduos que apresentaram resultados abaixo do resultado esperado para a faixa etária, em pelo menos um dos testes.

Todos os indivíduos, cujos resultados das avaliações não se encaixavam nos critérios de normalidade propostos para o estudo, foram encaminhados para atendimento com profissionais especializados. Orientações minuciosas quanto ao resultado das avaliações e estimulação auditiva foram fornecidas aos pais, professores e responsáveis pelas instituições na qual cada participante estava inserido, objetivando contribuir para o adequado atendimento e consequente melhora.

Para a realização das Avaliações Audiológicas Básicas e do Processamento Auditivo, fez-se necessário o uso de cabina acústica, otoscópio, imitanciômetro modelo 235h e audiômetro de dois canais AC40, ambos da Interacoustics, aparelho de CD player atrac3plus-Sony, Compact Disc (CD) de avaliação que acompanham o Manual de Avaliação do Processamento Auditivo Central, faixa 2 do $C D 1$ e faixa 3 do $C D 2$. Além do CD do teste Padrão de Duração - Faixa 4 e CD do teste RGDT, faixas 2, 3, 4,5 e 6 .

O projeto desta pesquisa foi aprovado pelo Comitê de Ética em Pesquisa da Faculdade de Ciências Médica da Universidade Estadual de Campinas - FCM/UNICAMP, sob parecer número 360/2005.

Visando verificar a existência de diferenças entre as variáveis categóricas em relação aos grupos foi aplicado o teste de McNemar corrigido pelo Qui-Quadrado, o qual compara dois grupos com relação às respostas dicotômicas. Para variáveis com três níveis de respostas foi utilizado o teste Qui-Quadrado de Stuart-Maxweel. Para as variáveis contínuas e ordenáveis foi utilizado o teste de Wilcoxon, objetivando verificar se os grupos eram diferentes ${ }^{14}$. $\mathrm{O}$ nível de significância adotado para os testes estatísticos foi de $5 \%$, ou seja, $p \leq$ 0,05 ; os quais foram indicados no texto por meio de asterisco $\left(^{*}\right)$.

\section{RESULTADOS}

$\mathrm{Na}$ Tabela 1 é apresentada a distribuição da amostra estudada, considerando-se o gênero, a faixa etária, além do diagnóstico oftalmológico de cegueira ou baixa visão e deficiência visual congênita ou adquirida para o Grupo I. Percebemos um número maior de crianças com baixa visão (10/15$66,6 \%)$ e deficiência visual congênita (13/15$86,6 \%$ ). Pode-se observar também que há um número maior de escolares do sexo masculino e na faixa etária de 8 a 10 anos.

$\mathrm{Na}$ Tabela 2 estão apresentados os resultados obtidos na avaliação audiológica básica para escolares do Grupo I e II, onde podemos observar a média de audibilidade para tons puros nas frequências de $500 \mathrm{~Hz}, 1000 \mathrm{~Hz}$ e $2000 \mathrm{~Hz}$ (X1), a média de audibilidade para tons puros nas frequências de $3000 \mathrm{~Hz}$ e $4000 \mathrm{~Hz}$ (X2), os Índices de Reconhecimento de Fala realizados com gravação (IRF*) e os tipos de Curvas Timpanométricas. Vale ressaltar que a via óssea do indivíduo número 3 encontrava-se dentro dos padrões de normalidade, assim como nos indivíduos de número 05 e 13, caracterizando perda auditiva do tipo condutiva. Já no indivíduo 12, estava acoplada a via aérea, indicando um comprometimento sensorioneural.

$\mathrm{Na}$ Tabela 3, observa-se a estatística descritiva em relação às médias de audibilidade obtidas para os Grupos I e II. Os escolares do Grupo I apresentaram limiares auditivos com valores maiores do que as do Grupo II, tanto para a orelha direita como 
Tabela 1 - Distribuição da amostra, considerando-se a faixa etária e o gênero masculino (M) e feminino (F), nos Grupos I e Il e o diagnóstico oftalmológico de cegueira e baixa visão (BV) e deficiência visual congênita (DV Congênita) ou adquirida (DV adquirida) para o Grupo I

\begin{tabular}{lcccccc}
\hline & \multicolumn{2}{c}{ Grupo I } & \multicolumn{2}{c}{ Grupo II } & \multicolumn{2}{c}{ Total } \\
\hline & $\mathbf{N}$ & $\%$ & $\mathbf{N}$ & $\%$ & $\mathbf{N}$ & $\%$ \\
\hline $\mathbf{8}$ & 9 & 30 & 9 & 30 & 18 & 60 \\
$\mathbf{1 0} \mathbf{1 0}$ & 6 & 20 & 6 & 20 & 12 & 40 \\
Masculino & 11 & 36,6 & 11 & 36,6 & 22 & 73,3 \\
Feminino & 4 & 13,3 & 4 & 13,3 & 8 & 26,6 \\
Cegueira & 5 & 33,3 & 0 & $0 \%$ & 5 & 33,3 \\
Baixa Visão & 10 & 66,6 & 0 & $0 \%$ & 10 & 66,6 \\
& & & & & & \\
DV Congênita & 13 & 86,6 & 0 & $0 \%$ & 13 & 86,6 \\
DV Adquirida & 2 & 13,3 & 0 & $0 \%$ & 2 & 13,3 \\
\hline
\end{tabular}

DV: Deficiência Visual

Tabela 2 - Escolares do Grupo I e II, segundo os resultados da avaliação audiológica básica

\begin{tabular}{|c|c|c|c|c|c|c|c|c|c|c|c|c|c|c|c|}
\hline \multicolumn{16}{|c|}{ Avaliação Audiológica Básica } \\
\hline \multirow[t]{2}{*}{ G I } & & \multirow{2}{*}{$\begin{array}{c}\text { X1 } \\
\text { dBNA }\end{array}$} & \multirow{2}{*}{$\begin{array}{c}\text { X2 } \\
\text { dBNA }\end{array}$} & \multirow{2}{*}{$\begin{array}{c}\text { IRF* }^{*} \\
\%\end{array}$} & \multirow{2}{*}{$\begin{array}{l}\text { CT } \\
\text { Tipo }\end{array}$} & \multicolumn{2}{|c|}{$\begin{array}{c}\text { Reflexos } \\
\text { Acústicos }\end{array}$} & \multirow[t]{2}{*}{ G II } & & \multirow{2}{*}{$\begin{array}{c}\text { X1 } \\
\text { dBNA }\end{array}$} & \multirow{2}{*}{$\begin{array}{c}\text { X2 } \\
\text { dBNA }\end{array}$} & \multirow{2}{*}{$\begin{array}{c}\text { IRF* }^{*} \\
\%\end{array}$} & \multirow{2}{*}{$\begin{array}{l}\text { CT } \\
\text { Tipo }\end{array}$} & \multicolumn{2}{|c|}{$\begin{array}{c}\text { Reflexos } \\
\text { Acústicos }\end{array}$} \\
\hline & & & & & & Contra & Ipsi & & & & & & & Contra & Ipsi \\
\hline \multirow[t]{2}{*}{1} & OD & 6,6 & 5 & 100 & A & $P$ & $P$ & 1 & OD & -5 & 0 & 96 & $A$ & $P$ & $P$ \\
\hline & OE & 10 & 5 & 100 & A & $P$ & $P$ & & OE & $-3,3$ & 5 & 96 & $A$ & $P$ & $P$ \\
\hline \multirow[t]{2}{*}{2} & OD & 8,3 & 0 & 92 & A & $P$ & $P$ & 2 & OD & 8,3 & 5 & 100 & $A$ & $P$ & $P$ \\
\hline & OE & 6,6 & 2,5 & 100 & A & $P$ & $P$ & & $\mathrm{OE}$ & 10 & 10 & 96 & A & & $P$ \\
\hline \multirow[t]{2}{*}{3} & OD & 26,6 & 47,5 & NC & B & A & A & 3 & OD & 8,3 & 2,5 & 100 & A & $P$ & $P$ \\
\hline & $\mathrm{OE}$ & 53,3 & 110 & NC & B & A & A & & $\mathrm{OE}$ & 3,3 & 2,5 & 100 & A & $P$ & $P$ \\
\hline \multirow[t]{2}{*}{4} & OD & 3,3 & 0 & 96 & A & $P$ & $P$ & 4 & OD & 6,6 & 7,5 & 100 & A & $P$ & $P$ \\
\hline & $\mathrm{OE}$ & 1,6 & 2,5 & 96 & A & $P$ & $P$ & & $\mathrm{OE}$ & 8,3 & 2,5 & 96 & A & $P$ & $P$ \\
\hline \multirow[t]{2}{*}{5} & OD & 10 & 10 & 96 & B & A & A & 5 & OD & 8,3 & 2,5 & 100 & A & $P$ & $P$ \\
\hline & $\mathrm{OE}$ & 1,6 & 2,5 & 100 & B & A & A & & $\mathrm{OE}$ & 6,6 & 0 & 100 & A & $P$ & $P$ \\
\hline \multirow[t]{2}{*}{6} & OD & 5 & $-2,5$ & 96 & A & $P$ & $P$ & 6 & OD & 1,6 & 2,5 & 100 & $A$ & $P$ & $P$ \\
\hline & $\mathrm{OE}$ & 5 & 2,5 & 96 & A & $P$ & $P$ & & $\mathrm{OE}$ & 1,6 & 5 & 100 & A & $P$ & $P$ \\
\hline \multirow[t]{2}{*}{7} & OD & 8,3 & 15 & 100 & A & $P$ & $P$ & 7 & OD & 6,6 & 2,5 & 96 & A & $P$ & $P$ \\
\hline & $\mathrm{OE}$ & 15 & 7,5 & 100 & A & $P$ & P & & $\mathrm{OE}$ & 6,6 & 0 & 100 & A & P & P \\
\hline \multirow[t]{2}{*}{8} & OD & 1,6 & 5 & 88 & A & $P$ & $P$ & 8 & OD & 3,3 & 0 & 100 & A & P & $P$ \\
\hline & $\mathrm{OE}$ & 0 & 2,5 & 88 & A & $P$ & $P$ & & $\mathrm{OE}$ & 3,3 & 0 & 100 & A & $P$ & $P$ \\
\hline \multirow[t]{2}{*}{9} & OD & 8,3 & 0 & 92 & A & P & P & 9 & OD & 5 & $-2,5$ & 100 & A & $P$ & P \\
\hline & $\mathrm{OE}$ & 5 & 0 & 100 & A & $P$ & $P$ & & $\mathrm{OE}$ & 3,3 & 0 & 96 & A & $P$ & $P$ \\
\hline \multirow[t]{2}{*}{10} & OD & 0 & 5 & 88 & A & P & P & 10 & OD & 1,6 & 0 & 100 & A & $P$ & P \\
\hline & $\mathrm{OE}$ & 1,6 & 0 & 100 & A & $P$ & $P$ & & $\mathrm{OE}$ & 6,6 & 0 & 100 & A & $P$ & $P$ \\
\hline \multirow[t]{2}{*}{11} & OD & -5 & -10 & 96 & A & $P$ & $P$ & 11 & OD & 3,3 & 0 & 96 & A & $P$ & $P$ \\
\hline & $\mathrm{OE}$ & $-1,6$ & 5 & 92 & A & $P$ & P & & OE & 6,6 & 0 & 100 & A & P & $\mathrm{P}$ \\
\hline \multirow[t]{2}{*}{12} & OD & 11,6 & 27,5 & 92 & A & $P$ & P & 12 & OD & $-1,6$ & 2,5 & 92 & A & $P$ & $P$ \\
\hline & OE & 18,3 & 27,5 & 96 & A & $P$ & $P$ & & $\mathrm{OE}$ & 1,3 & 5 & 96 & A & $P$ & P \\
\hline 13 & OD & 20 & 53,3 & 88 & B & A & $P$ & 13 & OD & 1,6 & 2,5 & 100 & A & $P$ & $P$ \\
\hline & $\mathrm{OE}$ & 17,5 & 50 & 92 & B & A & A & & OE & 1,6 & 5 & 100 & A & $P$ & $P$ \\
\hline 14 & OD & 10 & 12,5 & 100 & A & $P$ & A & 14 & OD & $-1,6$ & 0 & 96 & A & $P$ & $P$ \\
\hline & $\mathrm{OE}$ & 11,6 & 12,5 & 100 & A & $P$ & $P$ & & $\mathrm{OE}$ & $-3,3$ & -5 & 96 & A & P & $\mathrm{P}$ \\
\hline 15 & OD & 5 & 2,5 & 100 & A & $P$ & $P$ & 15 & OD & 10 & 12,5 & 96 & A & $P$ & $P$ \\
\hline & $\mathrm{OE}$ & 8,3 & 12,5 & 100 & A & $P$ & $P$ & & OE & 3,3 & 10 & 96 & A & $P$ & $P$ \\
\hline
\end{tabular}

OD: Orelha Direita $\quad$ OE: Orelha Esquerda NC: Não foi capaz de concluir o teste.

X1: Média de audibilidade para tons puros nas frequências de $500 \mathrm{~Hz}, 1000 \mathrm{~Hz}$ e $2000 \mathrm{~Hz}$,em dBNA(Nível de Audição).

X2: Média de audibilidade para tons puros nas frequências de $3000 \mathrm{~Hz}$ e $4000 \mathrm{~Hz}$, em dBNA(Nível de Audição).

IRF*: Índice de Reconhecimento de Fala realizado com gravação.

CT: Curva Timpanométrica P: Presente A: Ausente $\quad \mathrm{N}$ : Não S: Sim 
Tabela 3 - Valores descritivos em relação a X1 e X2, considerando-se os grupos I e Il e a orelha direita e esquerda

\begin{tabular}{cccccc|cccc}
\hline & & \multicolumn{4}{c|}{ Média X1 } & \multicolumn{4}{c}{ Média X2 } \\
\cline { 3 - 10 } & & Média & Mínimo & Máximo & p-valor & Média & Mínimo & Máximo & p-valor \\
\hline \multirow{2}{*}{ OD } & Grupo I & 8,00 & $-5,00$ & 26,67 & & 9,00 & $-10,00$ & 47,50 & \\
& Grupo II & 4,00 & $-1,67$ & 10,00 & 0,130 & 2,50 & $-2,50$ & 12,50 & 0,146 \\
\multirow{2}{*}{ OE } & Grupo I & 12,67 & $-1,67$ & 53,33 & & 16,17 & 0,00 & 110,00 & \\
& Grupo II & 3,56 & $-3,33$ & 10,00 & 0,163 & 1,33 & $-5,00$ & 10,00 & $0,018^{*}$ \\
\hline
\end{tabular}

Média X1: Média aritmética dos limiares auditivos das frequências de 500,1000 e $2000 \mathrm{~Hz}$

Média X2: Média aritmética dos limiares auditivos das frequências de 3000 e $4000 \mathrm{~Hz}$

para a orelha esquerda, considerando-se a média calculada para os limiares das frequências baixas e médias (X1) e para as frequências de 3000 e $4000 \mathrm{HZ}$ (X2). Para a média dos limiares de audibilidade calculada para as freqüências de 3000 e 4000 $\mathrm{Hz}$ na orelha esquerda, verifica-se que a diferença foi estatisticamente significante quando comparouse as respostas dos Grupos I e II.

Já na tabela 4, é possível observar os aspectos relacionados ao histórico escolar dos participantes de acordo com os dados obtidos em anamnese. Foram analisados achados relacionados a dificuldade na escrita, na leitura,presença de repetência escolar e o grau de escolaridade.
Todas as crianças do Grupo I e II estavam inseridas no sistema regular de ensino, sendo que no Grupo I, 10 (66,6\%) apresentaram repetência em algum momento. Quanto ao aprendizado da escrita e da leitura, há uma diferença estatisticamente significante entre os Grupos I e II apontada pela análise que mostrou mais dificuldades quando comparados aos participantes do Grupo II ( $p$-valor = $\left.0.031^{*}\right)$. A análise estatística também mostrou diferença significante entre os Grupos I e II em relação à repetência escolar $\left(p\right.$-valor $\left.=0.002^{*}\right)$.

Na Figuras 1 e tabelas 5, 6, 7, 8 e 9 apresentamos os resultados da bateria de testes comportamentais do Processamento Auditivo.

Tabela 04 - Escolares do Grupo I e II, com relação aos aspectos escolares, segundo os dados da anamnsese

\begin{tabular}{|c|c|c|c|c|c|c|c|}
\hline G I & $\begin{array}{c}\text { Dif. na } \\
\text { Escrita e } \\
\text { leitura }\end{array}$ & $\begin{array}{l}\text { Repetência } \\
\text { Escolar }\end{array}$ & $\begin{array}{c}\text { Grau de } \\
\text { Escolaridade }\end{array}$ & GII & $\begin{array}{c}\text { Dif. na } \\
\text { Escrita e } \\
\text { Leitura }\end{array}$ & $\begin{array}{l}\text { Repetência } \\
\text { Escolar }\end{array}$ & $\begin{array}{c}\text { Grau de } \\
\text { Escolaridade }\end{array}$ \\
\hline 1 & $\mathrm{~N}$ & $S$ & 6a série & 1 & $\mathrm{~N}$ & $\mathrm{~N}$ & 6a série \\
\hline 2 & $S$ & $S$ & Cl. Especial & 2 & $\mathrm{~N}$ & $\mathrm{~N}$ & $5 a$ série \\
\hline 3 & $S$ & $S$ & 2a série & 3 & $\mathrm{~N}$ & $\mathrm{~N}$ & 4a série \\
\hline 4 & $\mathrm{~N}$ & $S$ & 3a série & 4 & $\mathrm{~N}$ & $\mathrm{~N}$ & 4a série \\
\hline 5 & $S$ & $S$ & 3a série & 5 & $\mathrm{~N}$ & $\mathrm{~N}$ & 4a série \\
\hline 6 & $\mathrm{~N}$ & $\mathrm{~N}$ & 3a série & 6 & $\mathrm{~N}$ & $\mathrm{~N}$ & 3a série \\
\hline 7 & $\mathrm{~N}$ & $S$ & Cl. Especial & 7 & $\mathrm{~N}$ & $\mathrm{~N}$ & 2a série \\
\hline 8 & $\mathrm{~N}$ & $\mathrm{~N}$ & 3a série & 8 & $\mathrm{~N}$ & $\mathrm{~N}$ & 3a série \\
\hline 9 & $\mathrm{~N}$ & $\mathrm{~N}$ & 3a série & 9 & $\mathrm{~N}$ & $\mathrm{~N}$ & 3a série \\
\hline 10 & $\mathrm{~N}$ & $S$ & 4a série & 10 & $\mathrm{~N}$ & $\mathrm{~N}$ & 4a série \\
\hline 11 & $S$ & $S$ & 1a série & 11 & $\mathrm{~N}$ & $\mathrm{~N}$ & 4a série \\
\hline 12 & $S$ & $S$ & 3a série & 12 & $\mathrm{~N}$ & $\mathrm{~N}$ & 3a série \\
\hline 13 & $S$ & $\mathrm{~N}$ & 2a série & 13 & $\mathrm{~N}$ & $\mathrm{~N}$ & 3a série \\
\hline 14 & $\mathrm{~N}$ & $S$ & 3a série & 14 & $\mathrm{~N}$ & $\mathrm{~N}$ & 6a série \\
\hline 15 & $\mathrm{~N}$ & $\mathrm{~N}$ & 2a série & 15 & $\mathrm{~N}$ & $\mathrm{~N}$ & 2a série \\
\hline
\end{tabular}

Dificuldade na escrita e leitura : $\mathrm{Gl} \times$ Gll: $\mathrm{p}$-valor $=0.031$

Repetência escolar : Gl x Gll : p-valor $=0.002$. 


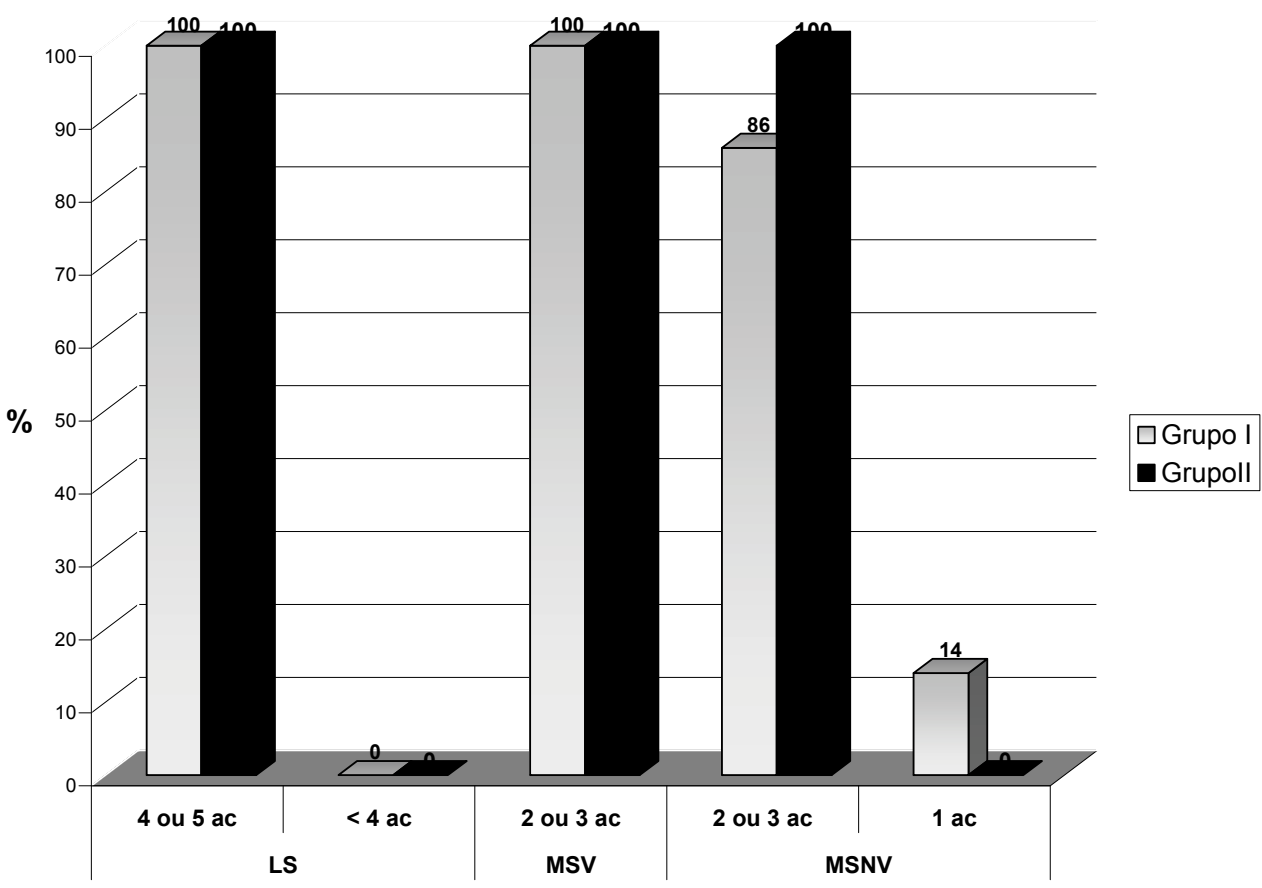

Figura 1 - Escolares do Grupo I e Grupo II, segundo o número de acertos (ac), em porcentagem, nos testes de Localização Sonora (LS), Memória Sequencial Verbal (MSV) e Memória Sequencial Não Verbal (MSNV)

Tabela 5 - Escolares dos Grupos I e II, segundo o menor intervalo de tempo percebido, em milisegundos (ms), no teste RGDT, para as frequências testadas (F)

\begin{tabular}{cccccccccccc}
\hline Freq & Grupo & $\mathbf{2}$ & $\mathbf{5}$ & $\mathbf{1 0}$ & $\mathbf{1 5}$ & $\mathbf{2 0}$ & $\mathbf{2 5}$ & $\mathbf{3 0}$ & $\mathbf{4 0}$ & $\mathbf{N C}$ & p-valor \\
\hline \multirow{2}{*}{$\mathbf{5 0 0 ~} \mathbf{~ H z}$} & I & 0 & 2 & 2 & 2 & 2 & 1 & 0 & 0 & 5 & \multirow{2}{*}{0,726} \\
& II & 0 & 4 & 5 & 3 & 3 & 0 & 0 & 0 & 5 & \\
$\mathbf{1 0 0 0} \mathbf{~ H z}$ & I & 1 & 0 & 4 & 0 & 2 & 0 & 2 & 0 & 5 & 0,181 \\
& II & 1 & 1 & 6 & 5 & 2 & 0 & 0 & 0 & 0 & \\
$\mathbf{2 0 0 0 ~ H z}$ & I & 0 & 2 & 4 & 0 & 1 & 1 & 0 & 1 & 5 & 0,348 \\
& II & 1 & 2 & 6 & 4 & 1 & 0 & 0 & 1 & 0 & \\
$\mathbf{4 0 0 0 ~ H z}$ & I & 0 & 2 & 4 & 0 & 1 & 1 & 0 & 1 & 5 & 0,607 \\
& II & 0 & 1 & 6 & 5 & 3 & 0 & 0 & 0 & 0 & \\
\hline
\end{tabular}

NC : Não Concluiu

Tabela 6 - Escolares do Grupo I e Grupo II, segundo a porcentagem de acertos e os valores estatísticos obtidos no teste Fala com Ruído

\begin{tabular}{|c|c|c|c|c|c|c|c|c|c|c|}
\hline \multirow{4}{*}{$\%$} & \multicolumn{10}{|c|}{ Fala com Ruído } \\
\hline & \multicolumn{4}{|c|}{ Grupo I $(n=14)$} & \multicolumn{4}{|c|}{ Grupo II $(n=15)$} & \multirow{2}{*}{\multicolumn{2}{|c|}{ TOTAL }} \\
\hline & \multicolumn{2}{|c|}{ OD } & \multicolumn{2}{|c|}{ OE } & \multicolumn{2}{|c|}{ OD } & \multicolumn{2}{|c|}{ OE } & & \\
\hline & $\mathbf{N}$ & $\%$ & $\mathbf{N}$ & $\%$ & $\mathbf{N}$ & $\%$ & $\mathbf{N}$ & $\%$ & $\mathbf{N}$ & $\%$ \\
\hline $100 \vdash 84$ & 8 & 57,1 & 7 & 50,0 & 6 & 40,0 & 6 & 40,0 & 27 & 46,5 \\
\hline $84 \longmapsto 68$ & 5 & 35,7 & 6 & 42,8 & 7 & 46,6 & 9 & 60,0 & 27 & 46,5 \\
\hline$<68$ & 0 & $0 \%$ & 0 & $0 \%$ & 2 & 13,3 & 0 & $0 \%$ & 2 & 3,4 \\
\hline NC & 1 & 7,1 & 1 & 7,1 & 0 & $0 \%$ & 0 & $0 \%$ & 2 & 3,4 \\
\hline Total & 14 & 100 & 14 & 100 & 15 & 100 & 15 & 100 & 58 & 100 \\
\hline
\end{tabular}

p-valor (GI x GII): OD= 0,103; $O E=0,421$ 
Tabela 7 - Escolares dos Grupos I e II, segundo a porcentagem de acertos e os valores estatísticos obtidos no Teste Dicótico de Dígitos, para as orelhas direita (OD) e esquerda (OE)

\begin{tabular}{|c|c|c|c|c|c|c|c|c|c|c|c|}
\hline \multirow{4}{*}{\multicolumn{2}{|c|}{$\%$}} & \multicolumn{10}{|c|}{ Teste Dicótico de Dígitos } \\
\hline & & \multicolumn{4}{|c|}{ Grupo I $(n=14)$} & \multicolumn{4}{|c|}{ Grupo II (n=15) } & \multirow{2}{*}{\multicolumn{2}{|c|}{ Total }} \\
\hline & & \multicolumn{2}{|c|}{ OD } & \multicolumn{2}{|c|}{ OE } & \multicolumn{2}{|c|}{ OD } & \multicolumn{2}{|c|}{ OE } & & \\
\hline & & $\mathbf{N}$ & $\%$ & $\mathbf{N}$ & $\%$ & $\mathbf{N}$ & $\%$ & $\mathbf{N}$ & $\%$ & $\mathbf{N}$ & $\%$ \\
\hline 100 & $\vdash 93,75$ & 4 & 28,5 & 5 & 35,7 & 12 & 80,0 & 11 & 73,3 & 32 & 55,1 \\
\hline 93,75 & $\vdash 87,50$ & 2 & 14,2 & 1 & 7,1 & 2 & 13,3 & 4 & 26,6 & 9 & 15,5 \\
\hline & : 87,50 & 2 & 14,2 & 2 & 14,2 & 1 & 6,6 & 0 & - & 3 & 5,1 \\
\hline & NC & 6 & 42,8 & 6 & 42,8 & 0 & - & 0 & - & 12 & 20,6 \\
\hline & Total & 14 & 100 & 14 & 100 & 15 & 100 & 15 & 100 & 58 & 100 \\
\hline
\end{tabular}

p-valor (GI x GII): OD=0,159

$(\mathrm{N}=08) \quad \mathrm{OE}=0,159$

Tabela 8 - Escolares, segundo a porcentagem de acertos e os valores estatísticos, no teste Padrão de Duração, orelhas direita (OD) e esquerda (OE)

\begin{tabular}{|c|c|c|c|c|c|c|c|c|c|c|}
\hline \multirow{4}{*}{$\%$} & \multicolumn{10}{|c|}{ Teste Padrão de Duração } \\
\hline & \multicolumn{4}{|c|}{ Grupo I $(n=14)$} & \multicolumn{4}{|c|}{ Grupo II $(n=15)$} & \multirow{2}{*}{\multicolumn{2}{|c|}{ Total }} \\
\hline & \multicolumn{2}{|c|}{ OD } & \multicolumn{2}{|c|}{ OE } & \multicolumn{2}{|c|}{ OD } & \multicolumn{2}{|c|}{ OE } & & \\
\hline & $\mathbf{N}$ & $\%$ & $\mathbf{N}$ & $\%$ & $\mathbf{N}$ & $\%$ & $\mathbf{N}$ & $\%$ & $\mathbf{N}$ & $\%$ \\
\hline $100 \vdash 85$ & 0 & $0 \%$ & 0 & $0 \%$ & 0 & $0 \%$ & 0 & $0 \%$ & 0 & $0 \%$ \\
\hline $85 \longmapsto 70$ & 1 & 7,1 & 1 & 7,1 & 0 & $0 \%$ & 0 & $0 \%$ & 2 & 3,4 \\
\hline $70 \longmapsto 55$ & 0 & $0 \%$ & 0 & $0 \%$ & 1 & 6,6 & 3 & 20,0 & 4 & 6,8 \\
\hline $55 \longmapsto 40$ & 2 & 14,2 & 1 & 7,1 & 3 & 20,0 & 1 & 6,6 & 7 & 12,0 \\
\hline$<40$ & 2 & 14,2 & 3 & 21,4 & 11 & 73,3 & 11 & 73,3 & 27 & 46,5 \\
\hline NC & 9 & 64,2 & 9 & 64,2 & 0 & $0 \%$ & 0 & $0 \%$ & 18 & 31,0 \\
\hline Total & 14 & 100 & 14 & 100 & 15 & 100 & 15 & 100 & 58 & 100 \\
\hline
\end{tabular}

p-valor (GI x GII): OD=0,172

$(\mathrm{N}=05) \quad \mathrm{OE}=0,24$

Tabela 9 - Escolares dos Grupos I e II, segundo a classificação normal e alterado para Avaliação Audiológica Básica (AAB)e do Processamento Auditivo(PA)

\begin{tabular}{|c|c|c|c|c|c|c|c|c|c|c|}
\hline & \multicolumn{4}{|c|}{ Avaliação Audiológica Básica } & \multicolumn{4}{|c|}{ Processamento Auditivo } & \multirow{2}{*}{\multicolumn{2}{|c|}{ Total }} \\
\hline & \multicolumn{2}{|c|}{ Normal } & \multicolumn{2}{|c|}{ Alterado } & \multicolumn{2}{|c|}{ Normal } & \multicolumn{2}{|c|}{ Alterado } & & \\
\hline & $\mathbf{N}$ & $\%$ & $\mathbf{N}$ & $\%$ & $\mathbf{N}$ & $\%$ & $\mathbf{N}$ & $\%$ & $\mathbf{N}$ & $\%$ \\
\hline Grupo I & 11 & 42,3 & 4 & 100 & 4 & 30,7 & 10 & 62,5 & 29 & 49,1 \\
\hline Grupo II & 15 & 57,6 & 0 & 0 & 9 & 69,2 & 6 & 37,5 & 30 & 58,8 \\
\hline Total & 26 & 100 & 4 & 100 & 13 & 100 & 16 & 100 & 59 & 100 \\
\hline
\end{tabular}

p-valor AAB (Grupo I x Grupo II)=0,125

p-valor PA (Grupo I x Grupo II)= 0,096

A partir desta etapa da avaliação da audição, torna-se imprescindível destacar que o fato de não concluir algum dos testes na bateria de processamentos auditivo, não implicou na obtenção de zero como score, mas sim, do participante ser excluído da análise estatística comparativa entre os Grupos I e II, o que diminui o número da amostra, pois alguns participantes do Grupo I não conseguiram concluir os testes Dicótico de Dígitos, Padrão de Duração e RGDT pela extrema dificuldade em responder a tarefa solicitada.

Assim sendo, não foi possível computar os dados de todos os participantes para que fosse realizada a análise estatística e comparativa entre os grupos 
nos testes Dicótico de Dígitos, Padrão de Duração e RGDT. Houve apenas, uma análise comparativa entre participantes que obtiveram algum score na sua realização, porém, ainda assim foi possível analisar dados relevantes sobre a audição de ambos os grupos.

Portanto, verifica-se que os escolares do Grupo I obtiveram resultados semelhantes aos escolares do Grupo II nos testes de Localização Sonora, Memória Seqüencial para Sons NãoVerbais e Fala com Ruído. Nos demais testes auditivos, envolvendo habilidades de processamento auditivo, os escolares do Grupo I apresentaram desempenho inferior ao Grupo II.

Ao questionarmos a relação existente entre comprometimento auditivo e diagnóstico oftalmológico, constatamos que os $04(26,6 \%)$ escolares do Grupo I com alteração na Avaliação Audiológica Básica eram portadores de baixa visão. Em relação ao Processamento Auditivo, dos escolares do Grupo I com comprometimento, 07 (46,6\%) eram portadores de baixa visão e 03 (20\%) eram cegos.

\section{DISCUSSÃO}

O presente estudo teve como principal objetivo analisar a audição de escolares portadores de deficiência visual (baixa visão e cegueira).

Com relação ao desempenho escolar, observouse que 10 (66,6\%) participantes do Grupo I, apresentaram repetência na sua carreira escolar, mesmo havendo o sistema de progressão continuada no Brasil. No entanto, esta idéia é compatível com estudos que apontam a deficiência visual como uma das possíveis causas de déficit na aprendizagem e conseqüente fracasso escolar. Sabe-se que os distúrbios do Processamento Auditivo também estão diretamente associados à repetência e ao fracasso escolar ${ }^{8}$ (Tabela 04).

$\mathrm{Na}$ avaliação audiológica básica constatou-se que no Grupo I, 4 (26,6\%) escolares obtiveram resultados alterados, sendo que $02(13,3 \%)$ apresentaram perda auditiva do tipo condutiva, 01 $(6,8 \%)$ perda mista e $01(6,85)$ sensórioneural Estudo da literatura apresentou resultados semeIhantes aos do presente estudo, com perda auditiva em $28 \%$ dos portadores de deficiência visual estudados ${ }^{15,16}$. Já no Grupo II todos os participantes obtiveram resultados de acordo com os padrões de normalidade propostos para o estudo. (Tabela 2)

$\mathrm{Na}$ análise das médias de audibilidade $\mathrm{X} 1$ (média de audibilidade para tons puros nas frequências de $500 \mathrm{~Hz}, 1 \mathrm{KHz}$ e $2 \mathrm{KHz}$ ) e X2 (média de audibilidade para tons puros nas frequências de $3 \mathrm{KHz}$ e $4 \mathrm{KHz}$ ), verificou-se que o Grupo II apresentou melhores níveis auditivos quando comparados com o Grupo
I. Os valores estatísticos para a média X2 diante a comparação do desempenho do Grupo I em relação ao Grupo II mostraram uma diferença estatisticamente significante em relação às respostas obtidas na orelha esquerda ( $p$-valor: $0,018^{*}$ ) (Tabela 3 ).

Estes dados vêem reforçar a importância da avaliação auditiva em crianças com deficiência visual, objetivando conhecer e aproveitar ao máximo o sentido da audição, já que nesta população o canal auditivo torna-se ainda mais importante como via de aprendizagem e conhecimento do mundo. A investigação e prevenção de alterações auditivas são de suma importância para o pleno desenvolvimento da criança portadora de deficiência visual. Vale salientar que por meio da audição, a pessoa com deficiência visual recebe uma variedade de sons informativos que caracterizam os mais diversos ambientes, dando pistas importantes sobre os lugares por onde percorre, assim como informa as direções a serem tomadas durante a sua trajetória. Além disso, para deficientes visuais graves e cegos, a audição é de especial importância, pois proporciona as informações que deveriam ser recebidas pelo sistema visual; serve de meio para orientação e mobilidade; proporciona dados para uma atuação independente no ambiente ${ }^{17}$.

Diante da bateria de testes do Processamento Auditivo, no teste de Localização Sonora para Cinco Direções, observamos que o Grupo I apresentou resultados semelhantes em comparação ao Grupo II (Figura 1). Estes dados corroboram com os achados de Dias e Pereira em estudo brasileiro que comparou o desempenho na lateralização de sons entre deficientes visuais e videntes, tendo sido verificado desempenho normal nos deficientes visuais ${ }^{18}$.

Nos testes Memória Sequencial Verbal e Memória Sequencial Não Verbal, percebe-se que o Grupo I esteve em homogeneidade ao Grupo II para o teste de Memória Sequencial Verbal, porém mostrou desempenho desfavorável em relação ao Grupo II no teste Memória Sequencial Não Verbal (Figura 1). As crianças do grupo I tiveram mais dificuldade em memorizar sons não-verbais.

Existem dois sistemas de memória separados, podendo funcionar de forma relativamente independente um do outro, a memória de curto prazo e a memória de longo prazo. A primeira referese à memória para eventos que ocorreram em um passado recente (acabaram de ocorrer), no qual o tempo entre a apresentação do material a ser lembrado e sua recordação não passa de alguns segundos ou minutos. Já a memória de longa duração se refere a eventos que ocorreram há horas, dias, meses ou até anos atrás. Estes dois sistemas de memória se desenvolvem, em 
termos de maturação do sistema nervoso, durante a infância, atingindo parâmetros semelhantes ao dos adultos por volta dos 10 a 12 anos de idade. Crianças de quatro anos de idade conseguem recordar sequências sonoras de dois a três itens, enquanto que crianças de 12 anos são capazes de recordar seis itens sonoros em sequência. A tarefa de recordação dos sons em sequência seria função do sistema de memória de curta duração. A capacidade de memória infantil tem importantes conseqüências para o aprendizado da escrita e que, crianças com prejuízos específicos de linguagem, geralmente apresentam uma habilidade pobre de memorização ${ }^{19}$.

No teste Fala com Ruído, o Grupo I apresentou resultados semelhantes em comparação ao Grupo II. A análise estatística nos indicou não haver diferença significante entre o desempenho das orelhas direita e esquerda nos grupos I e II (Figura 3).

Hipotetiza-se que o desempenho semelhante entre os Grupos ocorreu pela facilidade de repetição de palavras, mesmo com a presença de ruído de fundo, encontrada nas crianças com deficiência visual. O deficiente visual pode com facilidade usar palavras vazias de conteúdo sobre algo que jamais viu, como os nomes de cores, ou que o tato não pode abarcar, por ser pequeno ou grande demais. Na mesma linha, em testes de ordem superior, relacionados à utilização da linguagem, tais como "responder a indicações verbais" ou "imitar palavras", as crianças cegas obtêm excelentes resultados ${ }^{20}$.

No teste Dicótico de Dígitos, verificou-se um desempenho bastante inferior do Grupo I em relação ao Grupo II, em que seis participantes não conseguiram concluir a tarefa proposta e, se quer puderam ser computados na análise estatística (Figura 3). Este teste indica habilidade para agrupar componentes do sinal acústico em figura-fundo e identificá-los verbalmente. O processamento correto na orelha esquerda indica uma correta comunicação inter-hemisférica em nível de corpo caloso e resultados alterados em ambas as orelhas sugerem alterações no hemisfério esquerdo ${ }^{21}$. Desta forma, hipotetiza-se que as crianças com deficiência visual podem apresentar déficits na área de associação auditiva (relação inter-hemisférica, envolvendo corpo caloso) e/ou no hemisfério esquerdo.

Observando os testes Padrão de Duração e RGDT, constata-se uma diferença importante entre os grupos. No teste RGDT 05 (35,7\%) escolares do Grupo I não conseguiram concluir a tarefa e no Padrão de Duração, 09 (64,2\%) escolares também não concluíram (Figura 4 e Tabela 5). Estes testes envolvem processamento temporal auditivo, que se refere à percepção de um evento sonoro ou de uma alteração no mesmo, dentro de um intervalo definido de tempo ${ }^{22}$. O processamento temporal pode ser dividido em categorias que auxiliam no entendimento de alguns dos mecanismos e processos do sistema nervoso auditivo central. Uma delas é a ordenação ou sequencialização temporal, que se refere ao processamento de múltiplos estímulos auditivos na sua ordem de ocorrência. Graças a esta habilidade, um indivíduo é capaz de discriminar a correta ordem de ocorrência dos sons. A resolução temporal é outra categoria, definida como a habilidade do sistema auditivo em detectar mudanças rápidas e bruscas no estímulo sonoro ou o menor intervalo de tempo necessário para discriminar entre dois estímulos acústicos ${ }^{23}$.

O processamento temporal está intimamente relacionado com a percepção da fala, ou seja, sua duração, intensidade e frequência, além de aspectos de linguagem. Muitas evidências sugerem que as habilidades do processamento temporal são à base do processamento auditivo, especificamente no que concerne à percepção de fala. $\mathrm{O}$ argumento que suporta esta proposição é que muitas características da informação auditiva são, de alguma forma, influenciadas pelo tempo ${ }^{24,25}$. Além disso, o padrão temporal refere-se à habilidade do indivíduo em reconhecer contornos acústicos. Indivíduos com dificuldade para reconhecer o contorno acústico têm dificuldade para extrair e utilizar os aspectos prosódicos da fala, tais como ritmo, acentuação e entoação. Diferenças na acentuação, pistas prosódicas como pausas e velocidade de fala permitem que o ouvinte identifique a palavra chave e determine o conteúdo semântico ${ }^{26}$. Uma das etiologias para os comprometimentos temporais diz respeito à existência de problemas de bases perceptuais, envolvendo o processamento temporal de estímulos auditivos, visuais e sensório-motores, quando apresentados de maneira rápida e em seqüência. Esta teoria tenta conciliar déficits auditivos e visuais por meio de uma única base, o processamento temporal ${ }^{27,28}$, o que em parte justifica a dificuldade dos deficientes visuais nos testes que envolvem processamento temporal

Feita a análise dos testes de processamento auditivo, pode-se concluir que as habilidades auditivas de memória seqüencial para sons não verbais, figura-fundo para sons verbais e identificação de padrões sonoros encontram-se mais comprometidas nos indivíduos do Grupo I. Esses indivíduos com distúrbio do processamento devem receber tratamento adequado, para que não ocorra um bloqueio sério na comunicação, aprendizagem e no desenvolvimento biopsicossocial. 
Ao relacionar perda auditiva e comprometimento visual, pode-se constatar que os 04 (100\%) escolares com perda auditiva do Grupo I eram portadores de baixa visão. Em relação aos escolares com distúrbio do Processamento Auditivo, do Grupo I, 07 (70\%) eram portadoras de baixa visão e $03(30 \%)$ eram cegas. Com isso, é possível afirmar que há um maior número de crianças com baixa visão portadoras de alteração auditiva associada, as quais requerem maior atenção e cuidado, haja vista a importância da integridade auditiva para o desenvolvimento adequado da linguagem.

A literatura afirma que existe uma maior prevalência de crianças cegas a apresentarem desempenho adequado na área auditiva expressiva da linguagem em detrimento das crianças com baixa visão. Acreditando que a privação visual pode não interferir de forma desfavorável na aquisição da linguagem, ao contrário refere que a criança utiliza o sistema auditivo adquirindo e desenvolvendo habilidades para detectar, discriminar e localizar os sons e das estruturas que embasam a produção da voz e a articulação da fala para a produção dos sons, suprindo assim a ausência do input visual ${ }^{29}$.

Acredita-se que especialmente os profissionais envolvidos na habilitação e reabilitação dos portadores da população em questão devem estar atentos não só ao sentido da visão e sua capacidade de exploração visual, como também ao sentido da audição; visando utilizar da maneira mais eficaz as vias auditivas como meio de acesso às informações e aprendizagem, seja para a alfabetização, orientação e mobilidade, entre outras áreas específicas do trabalho multidisciplinar. A avaliação de uma habilidade sensorial na ausência de outra vem sendo alvo de vários estudos na comunidade científica ${ }^{30}$.

Ainda restam muitas dúvidas sobre os reais parâmetros do processamento auditivo em crianças portadoras de deficiência visual. Sugere-se então, outras investigações sobre o tema em pesquisas futuras.

Até o presente, acredita-se na importância da avaliação auditiva tanto em seus aspectos quantitativos como qualitativos, visto que tal sentido tornase uma das principais vias de acesso a informações e aprendizagem para esta população e assim, necessita de sua integridade para o pleno desenvolvimento biopsicossocial.

\section{CONCLUSÃO}

A partir da análise dos resultados obtidos no estudo envolvendo escolares deficientes visuais (GI) em comparação aos escolares providos de visão normal (GII), pode-se concluir que o Grupo I mostrou-se com desempenho desfavorável em relação ao Grupo II tanto na avaliação audiológica básica, como na bateria de testes do Processamento Auditivo. O mesmo foi observado para o desempenho escolar, havendo no Grupo I maior número de repetências e dificuldades no aprendizado da leitura e escrita.

\begin{abstract}
Purpose: analyze the hearing of students presenting with visual deficiency (low vision and blindness). Method: evaluation of $30(100 \%)$ children, divided into two groups: Group I comprised 15 (50\%) children with visual deficiency; and Group II comprised 15 (50\%) children with normal vision, without hearing and/or education complaints. The patients underwent meatoscopy, liminal tonal audiometry, logoaudiometry and immitanciometry; in addition to sound location tests in five directions, verbal and non-verbal sequential memory, speech-in-noise tests, dichotic digit tests, duration standard behavior and random gap detection test (RGDT). Results: concerning the basic auditory evaluation in Group I, four $(26.6 \%)$ students showed results altered - two $(13,3 \%)$ with conductive hearing loss, one $(6,8 \%)$ with mixed and one $(6,8 \%)$ with neurosensorial hearing loss. Conversely, all participants in Group II showed normal results. In the sequence of auditory processing tests, 10 (66.6\%) students in Group I showed results altered and four (26.6\%) showed normal results. Conclusion: Group I demonstrated unfavorable performance in comparison with Group II, either in the basic auditory evaluation or in the sequence of auditory processing tests.
\end{abstract}

KEYWORDS: Hearing; Vision, Low; Blindness; Auditory Perception 


\section{REFERÊNCIAS}

1. American Speech-Language-Hearing Association. Central auditory processing: current status of research and aplications for clinical practice. Task force on central auditory processing consensus development. Am J Audiol. 1996; 5:41-54.

2. Moller C. Deafblindness: living with sensory deprivation. Lancet. 2003; 362:46-7.

3. Fontané-Ventura J. Déficit auditivo. Retraso en el habla de origen audígeno. Rev Neurol. 2005; 41(1):25-37.

4. Bellis Bellis TJ. Neuromaturation and Neuroplasticity of the Auditory System. In: Bellis TJ. Assessment and Management of Central Auditory processing Disorders in the Educational Setting From Science to Practice. Canada:Thomson Deliviar Learning; 2003. p 103-39.

5. Izquierdo MA, Oliver DL, Malmierca MS. Mecanismos de plasticidad (funcional y dependiente de actividad)en el cerebro auditivo adulto y en desarrollo. Rev Neurol 2009; 48(8):421-9.

6. Pereira LD. Sistema Auditivo e Desenvolvimento das Habilidades Auditivas. In: Ferreira L (org.). Tratado de Fonoaudiologia, São Paulo: Roca; 2004. 7. Pereira LD, Schochat E. Processamento auditivo central: manual de avaliação. São Paulo: Lovise; 1997.

8. Perissinoto J. Atuação fonoaudiológica com o bebê prematuro: acompanhamento do desenvolvimento. In: Andrade CRF. Fonoaudiologia em berçário normal e de risco. Série: Atualidades em Fonoaudiologia. vol 1. São Paulo: Lovise; 1996.p.129-48.

9. Veitzman S. Visão subnormal. Rio de Janeiro: Cultura Médica; 2000.

10. Lopes Filho O. Tratado de fonoaudiologia. São Paulo: Roca; 2004.

11. Musiek FE, Baran JA, Pinheiro ML. Duration pattern recognition in normal subjects and patients with cerebral and cochlear lesions. Audiology. 1990; 29:304-13.

12. Balen AS, Liebel G, Boeno MRM, Mottecy CM. Resolução temporal de crianças escolares. Rev. CEFAC. 2009; 11(1):52-61.

13. Schochat E, Rabelo C M, Sanfins MD. Processamento auditivo central: testes tonais de padrão de frequência e de duração em indivíduos normais de 7 a 16 anos de idade. Pró-Fono. 2000; 12(2):1-7.

14. Conover WJ. Practical nonparametric statistics. New York: John Wiley; 1971.

15. Nuck ME, Blood GW, Blood IM. Fluent and disfluent normal speakers' responses on a Synthetic Sentence Identification (SSI) task. J Commun Disord. 1987;20(2):161-9.
16. Silveira EGC, Barros ALS, Falcão RA, Lins RAB, Freitas LC. Perfil audiométrico dos indivíduos portadores de cegueira. Rev Fonoaudiol Bras. 2004; 4(1):1-4.

17. Cobo AD, Rodrigues MG, Bueno ST. Aprendizagem e deficiência visual. In: Martin MB, Bueno ST, organizadores. Deficiência visual: aspectos psicoevolutivos e educativos. Santos: São Paulo; 2003. p.129-43.

18. Dias TLL, Pereira LD. Habilidade de localização e lateralização sonora em deficientes visuais. Rev Soc Bras Fonoaudiol. 2008; 13(4):352-6.

19. Gindri G, Keske-Soares M, Mota HB. Memória de trabalho, consciência fonológica e hipótese de escrita. Pró-Fono Revista de Atualização Científica 2007; 19(3):313-22.

20. Ortega MPP. Linguagem e deficiência visual. In: Martin MB, Bueno ST, organizadores. Deficiência visual: aspectos psicoevolutivos e educativos. Santos: São Paulo; 2003.

21. Colella-Santos MF. Processamento auditivo central: teste dicótico de dígitos em indivíduos normais. [tese]. São Paulo (SP): Universidade Federal de São Paulo; 1998.

22. Musiek FE, Shinn JB, Jirsa R, Bamiou DE, Baran JA, Zaidan E. GIN (gaps in noise) test performance in subjects with confirmed central auditory nervous system involvement. Ear Hear. 2005; 26(6):608-18. 23. Zaidan E, Garcia AP, Tedesco MLF, Baran JA. Desempenho de adultos jovens normais em dois testes de resolução temporal. Pró-Fono. 2008; 20(1):19-24.

24. Samelli AG, Schochat E. The gaps-in-noise test: gap detection thresholds in normal-hearing young adults. Int J Audiol. 2008; 47(5):238-45.

25. Musiek F, Shinn J, Jirsa B, Bamiou D, Baran J, Zaidan E GION (Gaps-In-Noise) test performance in subjects with confirmed central auditory nervous system involvement. Ear hear 2005; 26:608-18.

26. Samelli AG, Schochat E. Processamento auditivo, resolução temporal e teste de detecção de Gap: revisão da Literatura. Rev. CEFAC. 2008; 10(3):369-77.

27. Rosen S, Manganari E. Is there a relationship between speech and nonspeech auditory processing in children with dyslexia? J Speech Lang Hear Res. 2001; 44(4):720-36.

28. Murphy CFB, Schochat E. Influência de paradigmas temporais em testes de processamento temporal auditivo. Pró-fono Rev de Atualização Científica 2007; 19(3):259-66. 
29. Meirelles B. Aquisição da linguagem em crianças deficientes visuais de zero a três anos de idade. [trabalho de conclusão de curso] Campinas (SP): Universidade Estadual de Campinas; 2006.
30. Campos CF, Cruz MS, Feniman MR, RichieriCosta A. Processamento auditivo em indivíduos com deficiência visual: relato de caso. Rev Soc Bras Fonoaudiol. 2004; 9(2):112-5.

doi: 10.1590/S1516-18462011005000027

RECEBIDO EM: 13/09/2009

ACEITO EM: 09/11/2010

Endereço para correspondência:

Maria Francisca Colella dos Santos

Centro de Estudos e Pesquisas em Reabilitação

Professor Doutor Paulo O.S. Porto/ Faculdade

de Ciências Médicas/Universidade Estadual de

Campinas

Rua Tessalia Vieira de Camargo, 126

Campinas - SP

CEP: 13083-970

E-mail: mfcolella@ fcm.unicamp.br 